\title{
DETECTING FLU OUTBREAKS BASED ON SPATIOTEMPORAL INFORMATION FROM URBAN SYSTEMS - DESIGNING A NOVEL STUDY
}

\author{
L. O. Grottenberg ${ }^{1}$, O. Njå ${ }^{1}$, E. Tøssebro ${ }^{2}$, G.S. Braut ${ }^{3}$, R. Tønnessen ${ }^{4}$, G. M. Grøneng ${ }^{5}$ \\ ${ }^{1}$ Department of Safety, Economics and Planning, Faculty of Science and Technology, University of Stavanger, Norway \\ ${ }^{2}$ Department of Electrical Engineering and Computer Science, Faculty of Science and Technology, University of Stavanger, Norway \\ 3 Stavanger University Hospital, Norway \\ ${ }^{4}$ Department of Influenza, Norwegian Institute of Public Health, Norway \\ ${ }_{5}^{5}$ Department of Infectious Diseases Epidemiology and Modelling, Norwegian Institute of Public Health, Norway
}

\section{Geo-information for disaster management}

\begin{abstract}
KEY WORDS: GIS, epidemiology, spatial epidemiology, urban information systems, seasonal influenza, infectious disease
\end{abstract} surveillance and control

\begin{abstract}
This paper explores the application of real-time spatial information from urban transport systems to understand the outbreak, severity and spread of seasonal flu epidemics from a spatial perspective. We believe that combining travel data with epidemiological data will be the first step to develop a tool to predict future epidemics and to better understand the effects that these outbreaks have on societal functions over time. Real-time data-streams provide a powerful, yet underutilised tool when it comes to monitoring and detecting changes to the daily behaviour of inhabitants.

In this paper, we describe and discuss the design of the geospatial project, in which we will draw upon data sources available from the Norwegian cities of Oslo and Bergen. Historical datasets from public transport and road traffic will serve as an initial indication of whether changes in daily transport patterns corresponds to seasonal flu data. It is expected that changes in daily transportation habits corresponds to swings in daily and weekly flu activity and that these differences can be measured through geostatistical analysis. Conceptually one could be able to monitor changes in human behaviour and activity in nearly true time by using indicators derived from outside the clinical health services. This type of more up-to-date and geographically precise information could contribute to earlier detection of flu outbreaks and serve as background for implementing tailor-made emergency response measures over the course of the outbreaks.
\end{abstract}

\section{INTRODUCTION}

The societal ability to respond to major outbreaks is heavily dependent on successful interactions between a wide series of participants, components and processes. Efficient response during the early stages of a flu outbreak requires an awareness of the initial impact and severity of flu, and is reliant on early detection mechanisms. Accomplishing this requires a major emphasis on having robust technological and organisational tools and methods available to manage information flow and decision support between stakeholders at all levels of the hierarchy (WHO, 2017a).

This paper describes the design of a project that seeks to examine the viability of monitoring real-time information from urban systems to detect the outbreak/significant increase in the occurrence of flu in urban societies. To manage seasonal flu outbreaks, public health officials and epidemiologists use information compiled from the National Surveillance System for Influenza at the Norwegian Institute of Public Health (NIPH). The Norwegian Syndromic Surveillance System (NorSySS) is one of the data sources used in this system. NorSySS collects diagnosis data from general practitioners (GPs) and out-of-hours primary care facilities. Influenza-like illness (ILI), ICPC2 code R80 (WICC, 1998), is an indicator for flu activity that is used in the routine monitoring of flu to assess weekly flu trends at a local, regional and national level. These data go through a complex loop to collect, organize and anonymize flu metrics, before resulting in datasets that are applied for public health and epidemic management efforts.
The NIPH publishes annual risk assessments of seasonal flu (NIPH, 2017b) at the beginning of each flu season. They also publish end-of-season summaries. NIPH estimated the 2016/2017 epidemic to cause an excess of 1700 deaths (NIPH, 2017c). This is mirrored at the global scale, with the WHO estimating $5-15 \%$ of the world's population being infected with the seasonal flu each year (WHO, 2017a), resulting in 300,000 to 650,000 deaths per year (Iuliano et al., 2017). The Spanish flu presents a dire example of the human costs of major flu outbreaks, with $20-50$ million deaths, equal to $3-5 \%$ of the world's population (Guan et al., 2010). While recent outbreaks have not seen same severity, the risk for more severe flu outbreaks remains.

\subsection{Health sector}

One of the main future challenges of the Norwegian health sector is to manage the impact of future flu outbreaks, both of seasonal and non-seasonal variants. A flu pandemic is stated to be one of the main societal threats facing the Norwegian society (DSB, 2015), while the seasonal flu each year affects the entire society during the winter months. Rapid response to flu outbreaks requires a major mobilisation of societal resources, and depends on the activation of large parts of the national and international disease management structures.

The 2014 national pandemic flu preparedness plan (HOD, 2014) outlines the roles and responsibilities of the various components of the Norwegian health management structure, as 
well as the contributions of auxiliary parts of the national emergency management domain. A recent examination of the risk and vulnerability within the Norwegian health sector (HOD, 2017) outlines the main challenges facing the public health emergency management domain in Norway. Seasonal and pandemic flu are the main concerns, with major accidents and disasters as the secondary and tertiary focus areas. An emphasis is placed on issues relating to information security and systems integrity, with comparatively little focus placed on management of information. Issues arising from the lack of clear routines and responsibilities relating to flow of information in major health-related disasters is only briefly mentioned.

The current surveillance systems are not able to provide complete data on the spread of flu in real-time. Typically the delay can be more than one week when relying upon clinical reports and laboratory information. Conceptually one could be able to monitor changes in human behaviour and activity in nearly real-time by using indicators derived from outside the clinical health services. Combining the spatial components of existing data streams with GIS-based data from additional sources adds to the spatial and temporal quality of produced information, adding to the geographic understanding of the evolving risk picture.

This type of more up-to-date and geographically precise information could be a helping tool in the day-to-day planning and management of the health services related to an upcoming outbreak. It could make the planning of needed staffing more efficient and it could guide prioritization of possibly scarce resources related to medical equipment as e.g. respirators and equipment for extracorporeal membrane oxygenation. During the swine flu pandemic in 2009-2010 some concern turned up related to available capacity of life supporting medical equipment.

\subsection{Impact of the seasonal and pandemic flu}

In the Pandemic influenza severity assessment (PISA), The World Health Organization (WHO, 2017b) describes the following key metrics to assess influenza severity: Transmissibility, Seriousness of disease, Impact. These metrics describes the spread of influenza within a population, the virus effect on the individual person, as well as the effect of the epidemic and pandemic on health-care services and societal systems. It can be used as a tool for global and national risk assessment of flu outbreaks.

The National Risk Assessment (DSB, 2015) quantifies the costs of a major pandemic in Norway, based on the flu metrics developed by the NIPH and the WHO. The Directorate for Civil Protection (DSB) claims the effects of a severe flu outbreak to be major implying a large to very large impact on the Norwegian society. The likelihood is estimated to be high, $1-$ $2 \%$ per year, based on historical data such as the Spanish flu (1918), the Asian flu (1957), Hong Kong flu (1968), and the Swine flu (2009) (Guan et al., 2010). The direct impact on life and health is estimated to 1.2 million people infected (25\% of the Norwegian population), with a mortality rate of $0.5 \%$ $(6,125)$, and $3 \%(35,000)$ requiring hospitalization. The negative effect on critical infrastructure and societal function is supposed to increase the losses, resulting in an overall death toll of around 8,000 persons. The socioeconomic effects primarily stems from disruptions to production, with large numbers of the work force out at any given time over the course of the outbreak.

The quality of the assessments must be taken into consideration, and we will claim that further analyses should be initiated. The epidemic and pandemic risk assessments are limited by a lack of mechanisms to acquire updated information on societal functions and integrity over the course of an outbreak. Without a way to assess the impact of flu outbreaks on society, these calculations will remain inaccurate.

Our proposed framework and research methodology must be seen as a contribution to better understanding of factors and mechanisms in flu patterns. Hence, it would present a way to map and monitor the societal effects of flu outbreaks at earlier stages, where knowledge is scarce. We think this could significantly improve the quality of information used in risk assessments of epidemic and pandemic flu.

\subsection{Recent outbreaks}

One natural extension of the experiences with recent outbreaks is the fact that large-scale epidemics cannot solely be understood through the epidemic lens, but requires understanding the implications on other domains, such as safety, security and society (Bowsher, Milner, \& Sullivan, 2016). The social and societal dynamics of urban outbreaks comparatively see less exploration within spatial epidemiology, and there are few established mechanisms to map the integrity

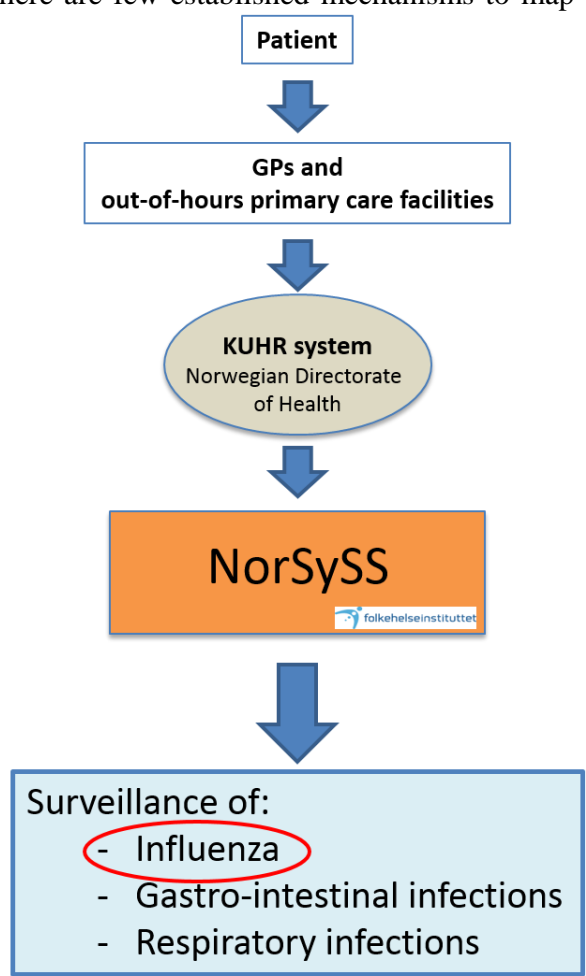

Figure 1: The Norwegian Syndromic Surveillance System (NIPH, 2017a) 
of societal systems and functions as outbreaks progress over time. The interconnectedness of modern sociotechnical systems creates a series of dependencies and links that is as of yet not understood, and there have been few outbreaks of sufficient severity to force the design and implementation of complex interdisciplinary information collection mechanisms. The role of up-to-date information from societal systems is at this point relatively unexplored and the emphasis has so far been on building capabilities within the respective institutions (Grottenberg \& Njå, 2017), rather than developing crossorganisational capabilities to collect, share and make use of information.

\section{NORWEGIAN FLU SURVEILLANCE STRUCTURE}

The NIPH carries the overall responsibility for monitoring influenza in Norway. The institute has a mandate to collect and manage information to monitor the occurrence and progress of flu outbreaks (MSIS, 2003), as well as to advice on public health matters resulting from effects of the seasonal flu. The flu monitoring system consists of six components (Table 1), with one being the NorSySS. NorSySS monitors reported cases of infectious diseases, including influenza, gastrointestinal and respiratory infections. The system provides an indicator-based surveillance capability for seasonal flu, based on reported flu cases from GPs and out-of-hours primary care facilities through the KUHR-system based at the Norwegian Directorate of Health. KUHR governs the control and payment of health reimbursements from the government.

Table 1: The Norwegian surveillance system for influenza

\begin{tabular}{|l|l|}
\hline System & Function \\
\hline NorSySS & $\begin{array}{l}\text { Indicator-based surveillance of } \\
\text { influenza-like illness in primary } \\
\text { health care }\end{array}$ \\
\hline $\begin{array}{l}\text { Hospital (all ward) } \\
\text { surveillance }\end{array}$ & $\begin{array}{l}\text { Laboratory-based surveillance of } \\
\text { hospitalised influenza cases }\end{array}$ \\
\hline ICU surveillance & $\begin{array}{l}\text { ICU treated flu patients. Data } \\
\text { collected by the Norwegian } \\
\text { Intensive Care Registry (pilot } \\
\text { project since 2016/17) }\end{array}$ \\
\hline Virological surveillance & $\begin{array}{l}\text { (1) Submission of data and } \\
\text { samples from Norwegian } \\
\text { laboratories testing for influenza. } \\
\text { (2) Sentinel system, GP-based } \\
\text { virological surveillance. }\end{array}$ \\
\hline $\begin{array}{l}\text { Norwegian mortality } \\
\text { monitoring system } \\
\text { (NorMOMO) }\end{array}$ & $\begin{array}{l}\text { Surveillance of weekly all-cause } \\
\text { excess mortality. }\end{array}$ \\
\hline $\begin{array}{l}\text { Seroepidemiological } \\
\text { analysis }\end{array}$ & $\begin{array}{l}\text { Annual survey of flu immunity in } \\
\text { the population. }\end{array}$ \\
\hline
\end{tabular}

NorSySS (NIPH, 2017a) receives data about the patient's age group, gender and the municipality of residence, the municipality where the consultation was performed, date and ICPC-2 diagnosis code (WICC, 1998). The datasets are compiled and anonymized through the KUHR system, before being passed on to NIPH and NorSySS for further analysis. ILI, ICPC-2 code R80, is used as an indicator for flu activity. The NIPH compiles weekly reports on flu surveillance during flu season (week 40 to week 20), where ILI \% is used to describe the occurrence of flu and the intensity of the outbreak compared with the intensity of previous outbreaks. The epidemic threshold for seasonal influenza (ILI \%) is based on the Moving
Epidemic Method (MEM) (Vega et al., 2013). These reports provides the public, health care workers and decision-makers with an understanding of the week-by-week development of the flu outbreak.

The data provided through the NorSySS has a high reliability, with data from nearly all GPs and out-of-hours primary care facilities in Norway and a firmly established routine and reporting methodology. The timeliness of the data is on the other hand lower, as most GPs accumulate the diagnosis data for a week or two before submitting to the KUHR system. This creates a situation where the datasets are not temporarily consistent, with some reports being submitted within a few days, while others lag by weeks. The lack of completeness is taken into account in the influenza surveillance by using the ratio of influenza consultations to the total number of consultations.

\section{SPATIAL CHARACTERISTICS OF AN URBAN OUTBREAK}

The spread of infectious diseases within an urban area can be predicted and modelled by the use of epidemiological tools. There have been multiple examinations that draw the link between human mobility and spread of diseases (Timpka et al., 2009), with development of complex models to account for urban and rural population patterns (Aleman, Wibisono, \& Schwartz, 2009; Poletto, Tizzoni, \& Colizza, 2013). The link between environment and health has been questioned by multiple authors (Robertson, 2017), demonstrating importance of modelling spatial environmental factors to accurately assess the spread of infectious diseases. Spread of infections within a societal system cannot be assumed to be static or uniform, but is rather a continuously changing process based on local factors, such as time of day, occupation, access to services and information patterns. The progress of an outbreak affects the movement patterns of inhabitants. Some measures of population self-control can be expected to be applied (Kleczkowski, Maharaj, Rasmussen, Williams, \& Cairns, 2015), making detailed pre-outbreak demographic data potentially outdated as outbreaks progress.

To accurately model the spread of disease in urban areas, it is necessary to understand the structure and dynamics within these societies. Yang, Pei, Chen, Liu, and Xia (2017) propose a framework to model the spatiotemporal characteristics of an outbreak, showcasing an approach to infer a dynamic social contact structure based on demographic data, heterogeneous surveillance data and epidemiological models. Yao et al. (2017) shows an application of multi-source geospatial data for modelling population distributions. Lewis and White (2017) demonstrates an approach that integrates multi-source surveillance information based on spatial components to model the incidence and prevalence rates of Chlamydia within a local population.

\section{A NOVEL STUDY FOR DETECTING FLU PATTERNS}

To augment the existing system, it is of interest to establish a monitoring system that enables immediate detection of emerging trends and patterns of influenza outbreaks, whether epidemic or pandemic. One possible way to build this understanding is to use the data produced within existing surveillance systems. Data streams from infrastructures provides interesting data that combines a high spatiotemporal resolution 


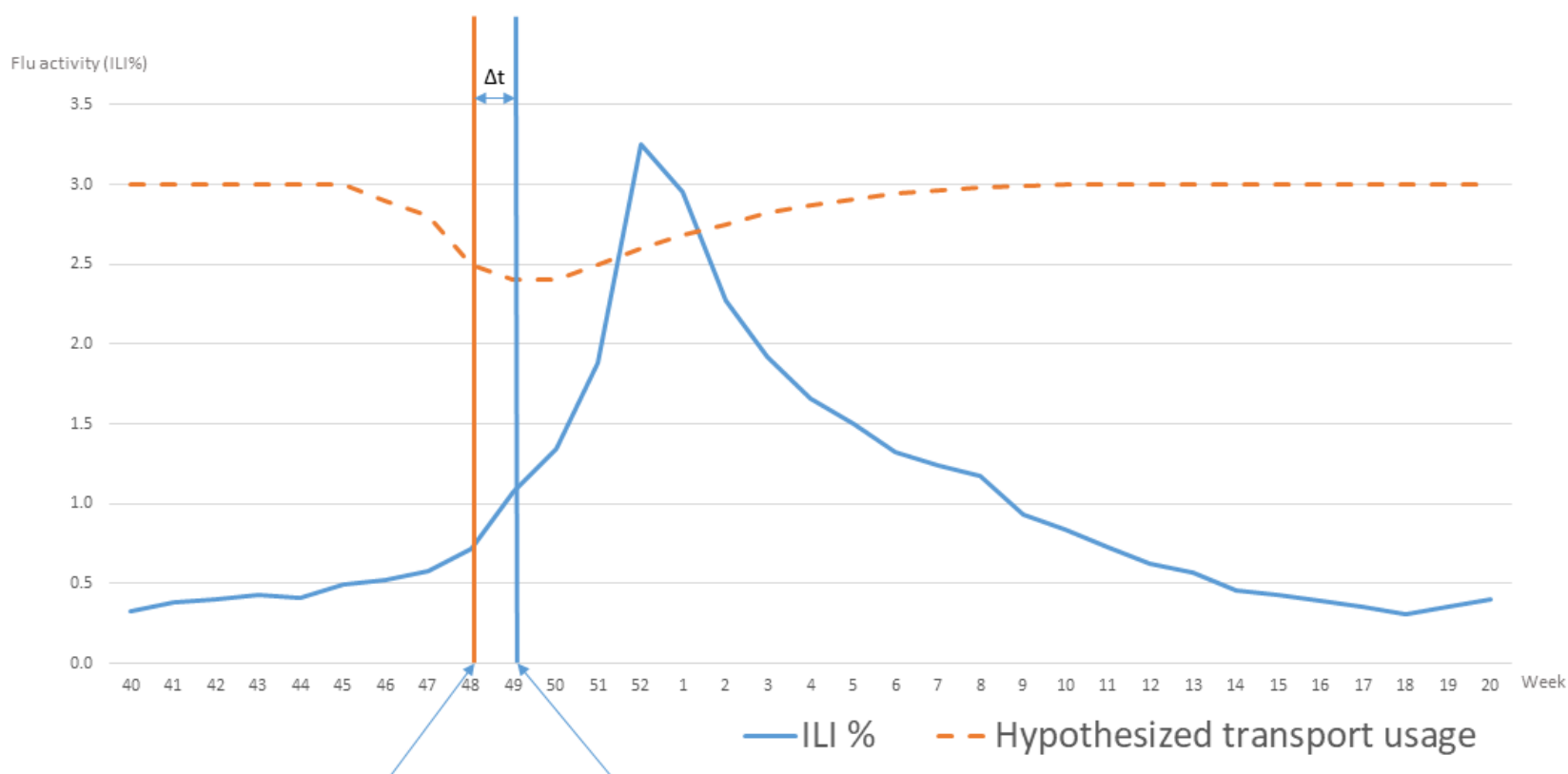

\section{Threshold for seasonal influenza based on transportation patterns \\ Epidemic threshold for seasonal influenza based on ILI \%}

Figure 2: Theoretical correlation between weekly public transportation utilisation and flu activity (ILI \%) in an urban population.

with frequent update schedules. Public transport, power utilisation, distribution of goods, and data traffic can be used as indicators of the day-to-day and hour-to-hour functioning of the societal systems that make up urban societies. The hypothesised interdependencies between usage of critical infrastructures and increased flu activity is illustrated in Figure 2. These interdependencies should be measurable in historic datasets on use patterns and flu activity, with increased flu activity being reflected in a decrease in daily use of public transport networks in major cities.

\subsection{Study approach}

Our goal is to augment the existing influenza surveillance system with an approach that monitors trends in society for indicators of flu activity. A search for similar works in the scientific literature at the thematic crossroads between epidemiological intelligence, spatial epidemiology and outbreak management did not reveal results.

The initial concept of an indicator-based seasonal flu monitoring system is presented to explore the role that spatial information and GIS can play in epidemiological intelligence. We believe that disruptions to the social patterns can be detected through spatial analysis on key metrics, such as daily travel patterns, consumption statistics and sales of key indicator goods.

\subsection{Preliminary hypotheses}

We need a research design that can reveal metrics, which are somehow interrelated with occurrence of seasonal flu. In order to improve abilities for flu emergency management we hypothesize that we will be able to become aware of the flu outbreak $\Delta \mathrm{t}$ prior to the normal identification of a flu outbreak, Figure 2 shows our hypothesized transport usage curve in a region combined with the actual clinically diagnosed influenza (ILI\%) curve in 2016/2017.

The first step of the study will focus on investigating and testing the two main hypotheses:

Hypothesis 1: A significant drop in commuting habits predicts seasonal flu outbreak.

Travel at specific times of day signifies ability to move between residential areas, work places, and leisure activities, and major disruptions to this ability should be traceable at daily level. To account for this, the transport-based monitoring systems will serve as a primary indicator for detecting changes in social patterns. Transport data will be augmented by other data sources, to strengthen the signal strength of patterns and eliminate sources of statistical noise that may falsely indicate pulses in flu impact.

The idea in this hypothesis is that changes in commuting habits fairly momentarily will detect seasonal flu. Such drops might be followed up with a more detailed investigation system. However the rate and absolute change will be interesting quantities to follow up in the study. We will also prepare the research on geospatial commuting data to explore severity levels and other characteristics of the seasonal flu.

Hypothesis 2: A significant increase in sales data on painkillers/antipyretics and antivirals predicts seasonal flu outbreaks.

In order to have a more robust system and even compare information we will investigate significant local changes in sales data of relevant pharmaceutical products, such as painkillers/antipyretics and antivirals. If sales metrics for these goods can be tied to pulses of the seasonal flu, this provides a 


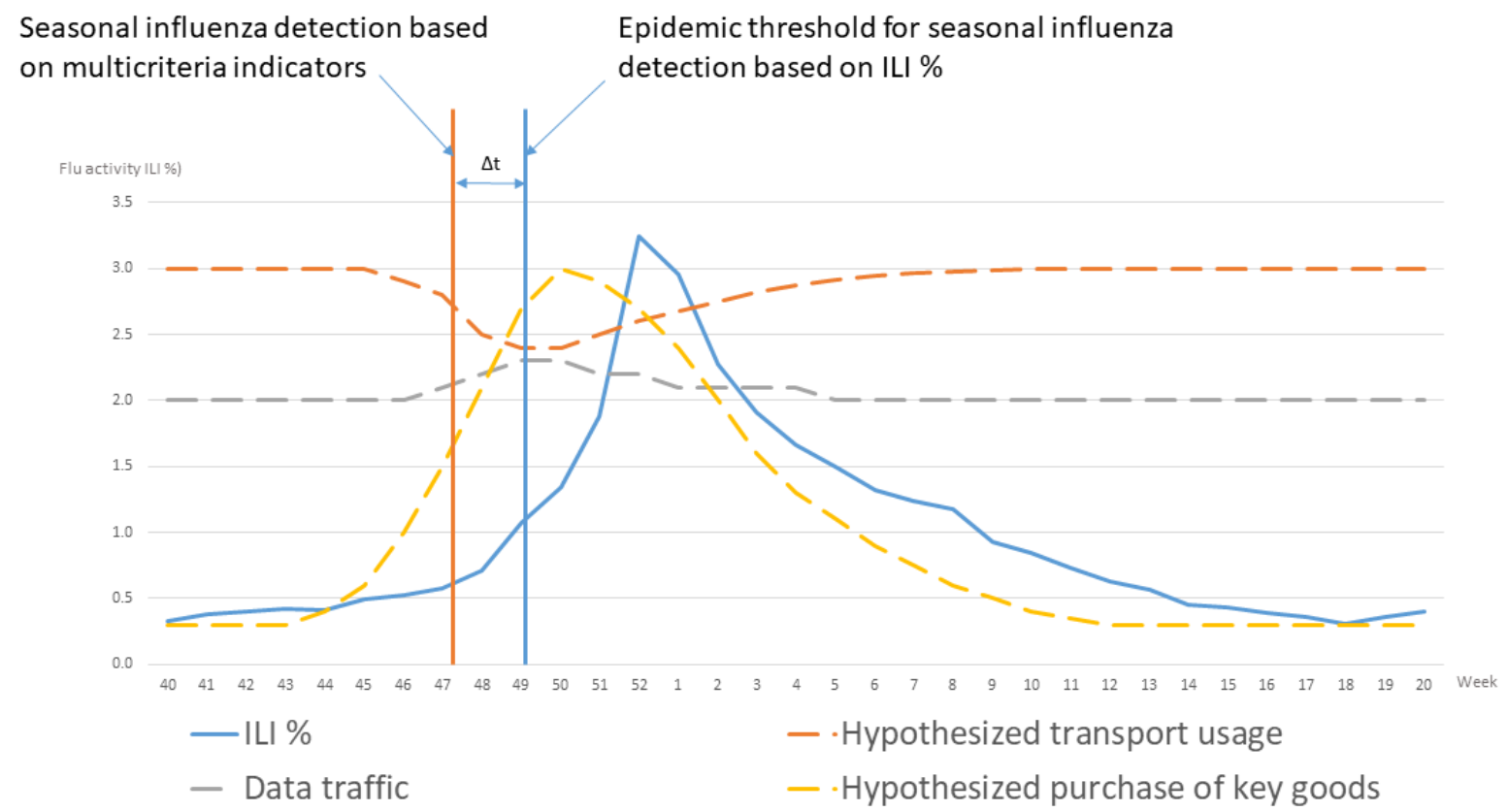

Figure 3: Multicriteria pattern detection based on indicator surveillance

powerful and readily available indicator that can be applied throughout the country.

We start with two fairly independent societal systems in which geospatial data is easy to retrieve and analyse. Further studies could implement other quantities, which could be historical datasets from public transport, road traffic data, as well as consumption-based metrics such as key indicator goods, utility usage and other available information sources from the cities of Bergen and Oslo. These data will be analysed for change patterns against the daily and weekly flu data delivered from NorSySS at the NIPH. The presence of detailed epidemiological datasets for Norwegian flu outbreaks will provide the necessary historical backdrop to demonstrate whether corresponding patterns and correlations between use of urban services and the presence of seasonal flu exist. We believe that it is possible to identify $\Delta \mathrm{t}$ when seen the data in retrospect and throughout some years develop a framework for seasonal flu surveillance.

\subsection{Proposed metrics}

The seasonal flu management within societies is relatively wellunderstood (Wolf, 2017), with clearly defined roles for the various actors in the civil society. For the outbreak management hierarchy, one of the key requirements is the availability of information that indicates the prevalence and impact of the flu outbreak in local societies. The dynamic nature of these phenomena lessens the role of static information sources, as the ground situation develops quickly over time. Applying these concepts to an information management perspective indicates a need for relevant information throughout all phases of an outbreak, to ensure that the stakeholders have the sufficient situational awareness.

To fill the present in the current-day intelligence networks, we propose an indicator-structure that integrates information from a variety of urban systems and critical infrastructure to maintain an up-to-date awareness of changes in patterns and trends within these systems. Table 2 highlights an initial set of indicators, based on the urban information systems available within major Norwegian cities. We believe that these indicators provide a way to detect possible trends at an early stage and provides a capability to compare daily and sub-daily disturbance against historical and current flu data. Use patterns of utilities, sales of key indicator goods (such as painkillers/antipyretics and antivirals), commercial activity and data traffic in residential areas provides powerful auxiliary information sources that are not mutually affected by events or incidents.

\section{Table 2: Urban systems indicators}

\begin{tabular}{|ll|}
\hline No & Indicator description \\
\hline $\mathbf{1}$ & $\begin{array}{l}\text { Public transport utilisation (Subway, trains, buses, light } \\
\text { rail, etc.) }\end{array}$ \\
\hline $\mathbf{2}$ & Toll road activations \\
\hline $\mathbf{3}$ & $\begin{array}{l}\text { Utility use patterns in residential and commercial areas } \\
\text { (Electricity, water, gas, etc.) }\end{array}$ \\
\hline $\mathbf{4}$ & $\begin{array}{l}\text { Use of key urban services (pharmacies, schools, GP } \\
\text { offices, etc.) }\end{array}$ \\
\hline $\mathbf{5}$ & $\begin{array}{l}\text { Activity information from commercial stakeholders } \\
\text { (convenience stores, restaurants, etc.) }\end{array}$ \\
\hline $\mathbf{6}$ & $\begin{array}{l}\text { Consumption of key indicator goods (Painkillers, } \\
\text { Tamiflu, coughing medicine, etc.) } \\
\text { Data traffic (internet traffic, cell phone networks) }\end{array}$ \\
\hline $\mathbf{7}$ &
\end{tabular}

Changes in use patterns may be the result of a variety of reason - Public holidays, cold weather, or fluctuations in the gas price may drastically change the weekly and daily statistics from public transport. Similarly, the other indicators are influenced by internal and external factors that affects the day-to-day use of the monitored systems. To combat these uncertainties, specific indicator profiles must be developed by exploiting the spatial nature of the measured data. Combined, the set of indicators provide a statistical foundation for further epidemic analysis, as well as the necessary data for implementation of analytical 
models for epidemiological work. The data-driven approach further will allow for rapid development of new indicator profiles, and can be adapted to the varying information needs over the course of an outbreak.

An example of applying trends from multiple urban indicators can be seen in Figure 3. By monitoring changes in multiple systems, in this case transport usage, purchase of key goods, and data traffic, it may be possible to detect changes to regular use patterns an earlier stage than in existing surveillance systems. This would move the $\Delta \mathrm{t}$ for flu detection forward, increasing the time and awareness for planning and management efforts in influenza outbreaks.

\subsection{Towards the development of a future framework}

By extending and formalising the information available to public health officials and decision-makers, the situational awareness of the impact and development of the seasonal flu can be maintained over time. The initial step of this process is to build the tools needed to maintain a data-driven awareness of societal functions in urban areas, based on the integration of data-sources with a high spatiotemporal quality. Figure 4 shows the general processes of a framework that implements the proposed indicator-based monitoring system, while Table 3 explores the processes, roles and responsibilities of this framework in more detail.

The benefits of such an approach is threefold - (1) Models of flu spread can be developed based on a broader set of information, (2) Secured access to information across the hierarchies of management organisations allows for increasingly collaborative systems and workspaces, (3) The societal effects of a seasonal or pandemic flu can be mapped in near real-time.

Access to updated information is a fundamental requirement to accomplish a multisector and multidisciplinary approach to health disaster management, as outlined in the Pandemic Influenza Risk Management (WHO, 2017a). The proposed system would offer a method for integration information from urban systems into decision-making processes in health-based disasters, and would fill one of the key requirements in a future comprehensive system of emergency management for health disasters.

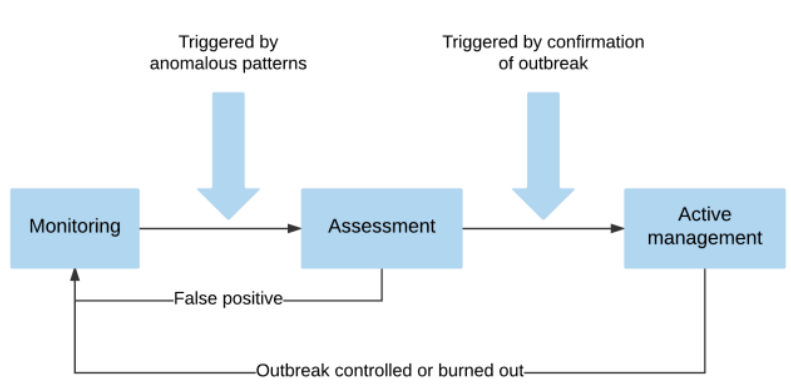

Figure 4: Process structure for indicator-based monitoring system

\section{CONCLUSION}

This paper outlines the design of an ongoing project to assess the suitability of data-streams from urban systems and critical infrastructures for epidemiological surveillance of flu outbreaks. It is our belief that real-time information from urban systems can fill an important role in improving the understanding of societal functions during major disasters and crises, and that multisource spatial information from urban systems, critical infrastructure and sector authorities can be combined with epidemiological information to provide actionable intelligence for use in influenza management efforts.

Pulses of seasonal flu should be directly traceable in historical data on public transport utilisation in major urban areas, with increases in flu activity corresponding to a drop in the utilisation of these service. Similar trends should also be present in data from other urban systems, providing a broad data-driven understanding of societal functions relating to management of infectious diseases.

Table 3: Outbreak process structure

\begin{tabular}{|lllll|}
\hline & Monitoring & Assessment & Initiation of management efforts \\
\hline $\begin{array}{l}\text { Outbreak } \\
\text { status }\end{array}$ & $\begin{array}{l}\text { Discrepancy in use } \\
\text { patterns }\end{array}$ & Outbreak suspected & Outbreak confirmed & Outbreak ongoing \\
\hline $\begin{array}{l}\text { Information } \\
\text { collection } \\
\text { methods }\end{array}$ & $\begin{array}{l}\text { Continuous } \\
\text { automated } \\
\text { monitoring of use } \\
\text { patterns }\end{array}$ & $\begin{array}{l}\text { Automated information } \\
\text { collected augmented by } \\
\text { manual collection }\end{array}$ & $\begin{array}{l}\text { Automated information } \\
\text { collected augmented by } \\
\text { manual collection }\end{array}$ & $\begin{array}{l}\text { Continuous impact } \\
\text { assessment }\end{array}$ \\
\hline $\begin{array}{l}\text { Activation } \\
\text { analytical } \\
\text { capacity }\end{array}$ & $\begin{array}{l}\text { Automated } \\
\text { analytical process }\end{array}$ & $\begin{array}{l}\text { Activation of manual } \\
\text { analysis capacity }\end{array}$ & $\begin{array}{l}\text { Deployment of } \\
\text { epidemiological } \\
\text { intelligence resources }\end{array}$ & $\begin{array}{l}\text { Participation of } \\
\text { national/transnational } \\
\text { analical resources }\end{array}$ \\
\hline $\begin{array}{l}\text { Epidemiologica } \\
\text { l work }\end{array}$ & $\begin{array}{l}\text { Mitigation and } \\
\text { monitoring }\end{array}$ & $\begin{array}{l}\text { Monitoring and notification } \\
\text { of key stakeholders }\end{array}$ & $\begin{array}{l}\text { Activation of response } \\
\text { apparatus }\end{array}$ & $\begin{array}{l}\text { Epidemiological response } \\
\text { Containment }\end{array}$ \\
\hline $\begin{array}{l}\text { Societal } \\
\text { activation }\end{array}$ & $\begin{array}{l}\text { No activation of } \\
\text { resources, day-to- } \\
\text { day activity }\end{array}$ & $\begin{array}{l}\text { Mobilisation of top-level } \\
\text { analytical resources }\end{array}$ & $\begin{array}{l}\text { Mobilisation of } \\
\text { specialist domain }\end{array}$ & Care/treatment \\
\hline
\end{tabular}




\section{REFERENCES}

Aleman, D. M., Wibisono, T. G., \& Schwartz, B. (2009, 13-16 Dec. 2009). Accounting for individual behaviors in a pandemic disease spread model. Paper presented at the Proceedings of the 2009 Winter Simulation Conference (WSC).

Bowsher, G., Milner, C., \& Sullivan, R. (2016). Medical intelligence, security and global health: the foundations of a new health agenda. Journal of the Royal Society of Medicine, 109(7), 269-273. doi: $10.1177 / 0141076816656483$

DSB. (2015). National Risk Assessment - 2014.

Grottenberg, L. O., \& Njå, O. (2017). Applying a systems safety approach to the development of GIS in the Norwegian emergency management domain. In Safety and Reliability - Theory and Applications (pp. 484-484): CRC Press.

Guan, Y., Vijaykrishna, D., Bahl, J., Zhu, H., Wang, J., \& Smith, G. J. D. (2010). The emergence of pandemic influenza viruses. Protein \& Cell, 1(1), 9-13. doi:10.1007/s13238-010-0008-z

HOD. (2014). National management plan for pandemic flu. The Norwegian Directorate of Health,

HOD. (2017). Overall risk and vulnerability routines in the healthcare sector. The Norwegian Directorate of Health,.

Iuliano, A. D., Roguski, K. M., Chang, H. H., Muscatello, D. J., Palekar, R., Tempia, S., . . Mustaquim, D. (2017). Estimates of global seasonal influenza-associated respiratory mortality: a modelling study. The Lancet. doi:10.1016/S0140-6736(17)33293-2

Kleczkowski, A., Maharaj, S., Rasmussen, S., Williams, L., \& Cairns, N. (2015). Spontaneous social distancing in response to a simulated epidemic: a virtual experiment. BMC Public Health, 15(1), 1-13. doi:10.1186/s12889-015-2336-7

Lewis, J., \& White, P. J. (2017). Estimating Local Chlamydia Incidence and Prevalence Using Surveillance Data. Epidemiology (Cambridge, Mass.), 28(4), 492-502. doi:10.1097/EDE.0000000000000655

Forskrift om Meldingssystem for smittsomme sykdommer (MSIS-forskriften). (2003).

NIPH. (2017a). About the Norwegian Syndromic Surveillance System.

NIPH. (2017b). Early risk assessment: What to expect of the $2017 / 18$ influenza season in Norway.

NIPH. (2017c). Influenza season in Norway 2016-17.

Poletto, C., Tizzoni, M., \& Colizza, V. (2013). Human mobility and time spent at destination: Impact on spatial epidemic spreading.

Robertson, C. (2017). Towards a geocomputational landscape epidemiology: surveillance, modelling, and interventions. GeoJournal, 82(2), 397-414.

Timpka, T., Eriksson, H., Gursky, E. A., Nyce, J. M., Morin, M., Jenvald, J., . . . Ekberg, J. (2009). Populationbased simulations of influenza pandemics: validity and significance for public health policy. Bulletin of the World Health Organization, 87(4), 305-311.

Vega, T., Lozano, J. E., Meerhoff, T., Snacken, R., Mott, J., Ortiz de Lejarazu, R., \& Nunes, B. (2013). Influenza surveillance in Europe: establishing epidemic thresholds by the Moving Epidemic Method. Influenza and Other Respiratory Viruses, 7(4), 546558. doi:10.1111/j.1750-2659.2012.00422.x

WHO. (2017a). Pandemic Influenza Risk Management.
WHO. (2017b). Pandemic Influenza Severity Assessment (PISA).

WICC. (1998). International Classification of Primary Care, ICPC-2. Oxford University Press, Oxford.

Wolf, M. (2017). Knowing Pandemics: An Investigation into the Enactment of Pandemic Influenza Preparedness in Urban Environments. Science \& Technology Studies, 30(4), 8-29.

Yang, B., Pei, H., Chen, H., Liu, J., \& Xia, S. (2017). Characterizing and Discovering Spatiotemporal Social Contact Patterns for Healthcare. IEEE Transactions on Pattern Analysis \& Machine Intelligence, $\quad 39(8), \quad$ 1532-1546. doi:10.1109/TPAMI.2016.2605095

Yao, Y., Liu, X., Li, X., Zhang, J., Liang, Z., Mai, K., \& Zhang, Y. (2017). Mapping fine-scale population distributions at the building level by integrating multisource geospatial big data. International Journal of Geographical Information Science, 31(6), 12201244. doi:10.1080/13658816.2017.1290252 